\title{
UNIQUENESS OF INVARIANT PRODUCT MEASURES FOR ELLIPTIC INFINITE DIMENSIONAL DIFFUSIONS AND PARTICLE SPIN SYSTEMS
}

\author{
Alejandro F. RAmírez ${ }^{1}$
}

\begin{abstract}
Consider an infinite dimensional diffusion process process on $T^{\mathbf{Z}^{d}}$, where $T$ is the circle, defined by the action of its generator $L$ on $C^{2}\left(T^{\mathbf{Z}^{d}}\right)$ local functions as $L f(\eta)=\sum_{i \in \mathbf{Z}^{d}}\left(\frac{1}{2} a_{i} \frac{\partial^{2} f}{\partial \eta_{i}^{2}}+b_{i} \frac{\partial f}{\partial \eta_{i}}\right)$. Assume that the coefficients, $a_{i}$ and $b_{i}$ are smooth, bounded, finite range with uniformly bounded second order partial derivatives, that $a_{i}$ is only a function of $\eta_{i}$ and that $\inf _{i, \eta} a_{i}(\eta)>0$. Suppose $\nu$ is an invariant product measure. Then, if $\nu$ is the Lebesgue measure or if $d=1,2$, it is the unique invariant measure. Furthermore, if $\nu$ is translation invariant, then it is the unique invariant, translation invariant measure. Now, consider an infinite particle spin system, with state space $\{0,1\}^{\mathbf{Z}^{d}}$, defined by the action of its generator on local functions $f$ by $L f(\eta)=\sum_{x \in \mathbf{Z}^{d}} c(x, \eta)\left(f\left(\eta^{x}\right)-f(\eta)\right)$, where $\eta^{x}$ is the configuration obtained from $\eta$ altering only the coordinate at site $x$. Assume that $c(x, \eta)$ are of finite range, bounded and that $\inf _{x, \eta} c(x, \eta)>0$. Then, if $\nu$ is an invariant product measure for this process, $\nu$ is unique when $d=1,2$. Furthermore, if $\nu$ is translation invariant, it is the unique invariant, translation invariant measure. The proofs of these results show how elementary methods can give interesting information for general processes.
\end{abstract}

Mathematics Subject Classification. 82C20, 82C22, 60H07, 60K35.

Received December 15, 2001.

\section{INTRODUCTION}

The mixing properties of general infinite dimensional processes are still poorly understood. In the particular case of infinite dimensional diffusions or infinite particle spin systems of local interactions and coordinates indexed by the hyper-cubic lattice $\mathbf{Z}^{d}$, most results concern the reversible case (see [3] for one of the few nonreversible results). In [5] and later in [8] and [7] it was proved that for a large class of these processes which need not to be reversible, in dimension $d=1$ and with compact state space, limit measures are invariant. If an additional uniqueness result for invariant measures is proved, this implies full convergence to the invariant measure for any initial distribution. Hence, it is natural to explore conditions under which such processes posses unique invariant measures. Let us remark that elliptic finite dimensional diffusions with compact state space always have a unique invariant measure. However, in infinite dimensions this is in general no longer true. In fact, it suffices to consider a diffusion process representing the dynamics of a spin system on the $d$-dimensional hyper-cubic lattice. When $d>1$, there might be more than one Gibbs state, which by construction are reversible with respect to the generator of the process. In this article we establish that for a large class of elliptic infinite

Keywords and phrases: Infinite dimensional diffusions, Malliavin calculus, Interacting particles systems.

${ }^{1}$ Facultad de Matemáticas, Pontificia Universidad Católica de Chile, Casilla 306, Correo 22, Santiago, Chile; e-mail: aramirez@mat.puc.cl 
dimensional diffusions and infinite particle spin systems, the existence of an invariant product measure implies its uniqueness. These results illustrate how to obtain interesting information about the mixing behavior of these processes by elementary means. These theorems have already been reported in [6].

Let us first consider infinite dimensional diffusions. Let $T$ be the unit circle. Consider $\Omega_{1}:=C\left([0, \infty) ; T^{\mathbf{Z}^{d}}\right)$ the space of continuous functions from $[0, \infty)$ to the space $T^{\mathbf{Z}^{d}}$, with the topology of uniform convergence in compact subsets of $[0, \infty)$. Consider the generator $L:=\sum_{i \in \mathbf{Z}^{d}}\left(\frac{1}{2} a_{i}(\eta) \frac{\partial^{2}}{\partial^{2} \eta_{i}}+b_{i}(\eta) \frac{\partial}{\partial \eta_{i}}\right)$, defined through its action on $C^{2}\left(T^{\mathbf{Z}^{d}}\right)$ local functions. Here $a, b: T^{\mathbf{Z}^{d}} \rightarrow \mathbf{R}^{\mathbf{Z}^{d}}$ are Borel-measurable, $a_{i}, b_{i}$ are their $i$-th coordinates, $\eta \in T^{\mathbf{Z}^{d}}$ and $\eta_{i}$ is the $i$-th coordinate of $\eta$. We say that the coefficients $a$ and $b$ are bounded if $\sup _{i, \eta}\left\{a_{i}, b_{i}\right\}<\infty$ and of finite range $R \in \mathbf{Z}^{+}$if for each $i \in \mathbf{Z}^{d}, a_{i}(\eta)$ and $b_{i}(\eta)$ depend only on coordinates $\eta_{j}$ of $\eta$ such that $|i-j| \leq R$. We say that the coefficients $a$ and $b$ have bounded second order partial derivatives if $\sup _{i, j, k}\left\{\frac{\partial^{2} a_{i}}{\partial \eta_{j} \partial \eta_{k}}, \frac{\partial^{2} b_{i}}{\partial \eta_{j} \partial \eta_{k}}\right\}<\infty$. The above generator with bounded, finite range coefficients $a$ and $b$ with bounded second order partial derivatives, defines a Feller semi-group $S_{t}$ on the space of continuous functions defined on $T^{\mathbf{Z}^{d}}$ [2]. Such a process will be called a finite range infinite dimensional diffusion family with bounded coefficients $a$ and $b$ with bounded second order partial derivatives. If $\inf _{i, \eta} a_{i}(\eta)<\infty$, we will say that this diffusion is uniformly elliptic.

In [7] it was shown in Theorem 4, using Lyapunov functional techniques similar to those developed by HolleyStroock [2], that given an infinite dimensional diffusion family with coefficients $\sigma$ and $b$ such that $\sigma_{i}=1, \frac{\partial b_{i}}{\partial \eta_{i}}=0$ and $b$ is smooth then the Lebesgue measure is the unique invariant measure. The first theorem is a considerable improvement of this result.

Theorem 1. Consider a finite range uniformly elliptic infinite dimensional diffusion family on $T^{\mathbf{Z}^{d}}$ with bounded coefficients $b$ and $\sigma$. Assume that they are smooth, have bounded second order partial derivatives and that $\sigma_{i}$ is a function only of $\eta_{i}$. Suppose that $\nu$ is a product measure which is invariant. Then,

(i) if $\nu$ is translation invariant, it is the unique invariant, translation invariant measure;

(ii) if $d=1$ or $2, \nu$ is the unique invariant measure;

(iii) if $\nu$ is the Lebesgue measure, it is the unique invariant measure.

Furthermore, if $d=1$ and $\mu$ is an arbitrary probability measure on $T^{\mathbf{Z}^{d}}$, then $\lim _{t \rightarrow \infty} \mu S_{t}=\nu$.

The last statement of this theorem is implied by part (ii) and Theorem 1 of [7]. Also, a simple consequence of part (iii) of the above theorem is the following corollary which gives a divergence free type condition which ensures that Lebesgue measure is invariant.

Corollary 1. Consider a finite range uniformly elliptic infinite dimensional diffusion family on $T^{\mathbf{Z}^{d}}$ with bounded coefficients $b$ and $\sigma$. Assume that they are smooth, have bounded second order partial derivatives and that $\sigma_{i}$ is a function only of $\eta_{i}$. Suppose there is an increasing sequence of finite subsets $\Lambda_{n} \subset \mathbf{Z}^{d}$ such that $\cup_{n=1}^{\infty} \Lambda_{n}=\mathbf{Z}^{d}$ and that $\lim _{n \rightarrow \infty} \int\left|\sum_{i \in \Lambda_{n}}\left(\frac{1}{2} \frac{\partial^{2} a_{i}}{\partial \eta_{i}^{2}}-\frac{\partial b_{i}}{\partial \eta_{i}}\right)\right| \mathrm{d} m=0$. Then the Lebesgue measure is the unique invariant measure of the diffusion process.

The second result of this article concerns infinite particle spin systems. Let $D:=\{0,1\}$. Consider the space $\Omega_{2}:=D\left([0, \infty) ; T^{\mathbf{Z}^{d}}\right)$ of right-continuous functions which have left-hand limits, endowed with the Skorohod topology. Consider the generator defined through its action on local function $f$ as $L_{2} f:=\sum_{x \in \mathbf{Z}^{d}} c(x, \eta)\left(f\left(\eta^{x}\right)-\right.$ $f(\eta))$. Here $\eta^{x} \in T^{\mathbf{Z}^{d}}$ is the configuration differing from $\eta$ only at site $x$ and $c(x, \eta): \mathbf{Z}^{d} \times D^{\mathbf{Z}^{d}} \rightarrow[0, \infty)$ is the jump rate at which $\eta$ changes to $\eta^{x}$. We will say that the jump rates are of finite range $R \in \mathbf{Z}^{+}$if for every $x \in \mathbf{Z}^{d}$, the function $c(x, \eta)$ depends only on coordinates $\eta_{y}$ of $\eta$ such that $|x-y| \leq R$. We will say that the jump rates are bounded if $\sup _{x, \eta} c(x, \eta)<\infty$. The above generator, with finite range and bounded jump rates, defines a Feller semi-group $S_{t}$ on the space of continuous functions defined on $D^{\mathbf{Z}^{d}}$ [4]. Such a process will be called a finite range infinite particle spin system with bounded flip rates. If in addition $\inf _{x, \eta} c(x, \eta)>0$, we say that this spin system is uniformly elliptic. 
Theorem 2. Consider a finite range uniformly elliptic infinite particle spin system on $D^{\mathbf{Z}^{d}}$ with bounded flip rates. Suppose that $\nu$ is a product measure which is invariant. Then,

(i) if $\nu$ is translation invariant, it is the unique invariant, translation invariant measure;

(ii) if $d=1$ or $2, \nu$ is the unique invariant measure.

Furthermore, if $d=1$ and $\mu$ is an arbitrary probability measure on $D^{\mathbf{Z}^{d}}$, then $\lim _{t \rightarrow \infty} \mu S_{t}=\nu$.

Note that the last statement is implied by Theorem 1 of Mountford [5] (see also [8]). The proof of parts $(i)$ and $(i i)$ are the discrete context version of parts $(i)$ and $(i i)$ of Theorem 1.

The proofs of Theorems 1 and 2 are based on an analysis of a Lyapunov functional related to a given invariant measure, which is computed on boxes $\Lambda_{n}$ converging to the whole lattice $\mathbf{Z}^{d}$ as $n \rightarrow \infty$. Showing that this functional vanishes is sufficient to prove a uniqueness result. The difficulty lies in the control of the boundary terms of the Lyapunov functional. An application of the method developed by Holley-Stroock [2] gives this control for $d \leq 2$, which in turn enables us to prove $(i i)$. A different kind of argument provides a short proof of $(i)$. The proof of $(i i i)$ in Theorem 1 is harder. The method we develop is inspired on the Lyapunov functional techniques of Holley-Stroock, but the crucial argument (Lem. 1) enabling us to pass to dimensions higher than 2 is of a different nature. This lemma shows that if Lebesgue measure is invariant, then the Lyapunov functional related to any other invariant measure should grow exponentially fast in boxes. This contradicts a polynomial bound which we can prove under mild hypothesis on the coefficients of the diffusion. The proof of the lemma involves a careful control of the boundary terms through the use of Poincaré inequality type estimates. It is here that this method fails when Lebesgue measure is replaced by an arbitrary product measure, although a weak enough dependence of the product measure on far sites would be enough.

In the first section of this article we will prove Theorem 1. Our first step will be to derive a crucial inequality, which when combined with a convexity property of the Lyapunov functional, will enable us to prove parts (i) and (ii) of the theorem in Sections 1.1 and 1.2 respectively. In Section 1.3 we prove part (iii) of Theorem 1, being the statement and proof of Lemma 1 the first step. In Section 2 we provide the proof of Theorem 2 . The main part of this proof is the derivation of an inequality analogous to the one proved for diffusions, which when combined with a convexity property of the corresponding Lyapunov functional, lets us prove the theorem. Here we follow closely the ideas of the proof of part (i) and (ii) of Theorem 1.

\section{INFINITE DIMENSIONAL DIFFUSIONS}

In this section we will prove Theorem 1. We will first introduce the notation that will be needed and then establish an inequality which will be the base for proving parts $(i, i i)$ and $($ iii $)$ of the theorem. So let $N \geq 1$ and $R$ the range of $b$ and define the boxes $\Lambda_{N}:=[-N R, N R]^{d} \cap \mathbf{Z}^{d}$ and its boundary $\delta \Lambda_{N}:=\Lambda_{N} \backslash \Lambda_{N-1}$. We also define $\Lambda_{N, i}:=\Lambda_{N} \cup B_{i}$, where $B_{i}:=\left\{j \in \mathbf{Z}^{d}:|i-j| \leq R\right\}$ is the ball of radius $R$ centered at site $i$. Given an arbitrary invariant measure $\mu$, and some finite subset $F \subset \mathbf{Z}^{d}$, we will denote by $u_{F}$ the RadonNykodim derivative of $\mu$ restricted to the subset $F$ with respect to the Lebesgue measure on $F$. We will call $u_{N}$ the Radon-Nykodim derivative $u_{\Lambda_{N}}$. Similarly we will denote by $v_{F}$ the Radon-Nykodim derivative of the given invariant measure $\nu$ restricted to $F$ and $v_{N}$ will stand for $v_{\Lambda_{N}}$. The existence and smoothness of these derivatives follows from Malliavin calculus (see for example Holley-Stroock). Let phi be some local smooth function depending on coordinates only on $\Lambda_{N}$. Then, since $\mu$ is invariant,

$$
0=\int \phi \frac{\partial u_{N}}{\partial t} \mathrm{~d} m=\sum_{i \in \Lambda_{N}} \int_{i} a_{i} \frac{1}{2} \frac{\partial^{2} \phi}{\partial \eta_{i}^{2}} u_{N} \mathrm{~d} m+\sum_{i \in \Lambda_{N}-1} \int_{i} b_{i} \frac{\partial \phi}{\partial \eta_{i}} u_{N} \mathrm{~d} m+\sum_{i \in \delta \Lambda_{N}} \int_{i} b_{i} \frac{\partial \phi}{\partial \eta_{i}} u_{\Lambda_{N, i}} \mathrm{~d} m
$$

Let us call $v_{i}$ the density of $\nu$ at site $i \in \mathbf{Z}^{d}$. Note that since $\nu$ is a product invariant measure, the measure on $T$ with density $v_{i}$ is invariant for the diffusion on $T$ defined by the generator $L_{i}:=\frac{1}{2} a_{i}(x) \frac{\mathrm{d}^{2}}{\mathrm{~d} x^{2}}+\bar{b}_{i}(x) \frac{\mathrm{d}}{\mathrm{d} x}$, where $\bar{b}_{i}(x):=\int b_{i}(\eta) \mathrm{d} \nu_{i}$ with $\eta_{i}=x$, and $\nu_{i}$ is the restriction of $\nu$ to $\mathbf{Z}^{d}-\{i\}$. By standard arguments for one dimensional diffusions, it follows from the smoothness of $a_{i}(x)$ and $\bar{b}_{i}(x)$ and the ellipticity, that $v_{i}$ is strictly 
positive on $T$. Compactness of $T \operatorname{implies~that~} \inf _{\eta} v_{i}(\eta)>0$ and therefore for each $N \geq 1$, $\inf _{\eta} v_{N}(\eta)>0$. By Theorem 2.25 of Holley-Stroock we can now conclude that for each $N \geq 1 \inf _{\eta} u_{N}(\eta)>0$. Hence, we can choose $\phi$ in (1) as the smooth function $\ln \frac{u_{N}}{v_{N}}$. Then (1) implies that,

$$
\begin{aligned}
0= & -\sum_{i \in \Lambda_{N}} \int a_{i} \frac{1}{2} \frac{1}{\left(u_{N} / v_{N}\right)^{2}}\left(\frac{\partial\left(u_{N} / v_{N}\right)}{\partial \eta_{i}}\right)^{2} u_{N} \mathrm{~d} m+\sum_{i \in \Lambda_{N}} \int a_{i} \frac{1}{2}\left(\frac{\partial^{2}}{\partial \eta_{i}^{2}} \frac{u_{N}}{v_{N}}\right) v_{N} \mathrm{~d} m \\
& +\sum_{i \in \Lambda_{N}-1} \int b_{i}\left(\frac{\partial}{\partial \eta_{i}} \frac{u_{N}}{v_{N}}\right) v_{N} \mathrm{~d} m+\sum_{i \in \delta \Lambda_{N}} \int b_{i} \frac{v_{N}}{u_{N}} \frac{\partial\left(u_{N} / v_{N}\right)}{\partial \eta_{i}} u_{\Lambda_{N, i}} \mathrm{~d} m .
\end{aligned}
$$

Now, let $v_{N, \delta, i}$ be the density of $\nu$ on $\Lambda_{N, i}-\Lambda_{N}$. Moving the first term of the left-most side to the right-most side of the above display, and adding and subtracting $\sum_{i \in \delta \Lambda_{N}} \int b_{i} \frac{1}{u_{N}} \frac{\partial\left(u_{N} / v_{N}\right)}{\partial \eta_{i}} u_{N} \mathrm{~d} \nu$ in the left hand side, we get

$$
\begin{aligned}
\sum_{i \in \Lambda_{N}} \int \frac{a_{i}}{2} \frac{v_{N}}{u_{N}}\left(\frac{\partial\left(u_{N} / v_{N}\right)}{\partial \eta_{i}}\right)^{2} v_{N} \mathrm{~d} m= & \sum_{i \in \Lambda_{N}} \int \frac{a_{i}}{2}\left(\frac{\partial^{2}}{\partial \eta_{i}^{2}} \frac{u_{N}}{v_{N}}\right) v_{\Lambda_{N, i}} \mathrm{~d} m+\sum_{i \in \Lambda_{N}} \int b_{i}\left(\frac{\partial}{\partial \eta_{i}} \frac{u_{N}}{v_{N}}\right) v_{\Lambda_{N, i}} \mathrm{~d} m \\
& +\sum_{i \in \delta \Lambda_{N}} \int b_{i} \frac{v_{N}}{u_{N}} \frac{\partial\left(u_{N} / v_{N}\right)}{\partial \eta_{i}}\left(u_{\Lambda_{N, i}}-u_{N} v_{\delta, N, i}\right) \mathrm{d} m \\
= & \sum_{i \in \delta \Lambda_{N}} \int b_{i} \frac{v_{N}}{u_{N}} \frac{\partial\left(u_{N} / v_{N}\right)}{\partial \eta_{i}}\left(u_{\Lambda_{N, i}}-u_{N} v_{\delta, N, i}\right) \mathrm{d} m
\end{aligned}
$$

where we have used the fact that $\nu$ is an invariant product measure which implies that $\sum_{i \in \Lambda_{N}} \int\left(\frac{a_{i}}{2} \frac{\partial^{2}}{\partial \eta_{i}^{2}} \frac{u_{N}}{v_{N}}+b_{i} \frac{\partial}{\partial \eta_{i}} \frac{u_{N}}{v_{N}}\right) v_{\Lambda_{N, i}} \mathrm{~d} m=0$. Now, since for each $i \in \mathbf{Z}^{d}$, the function $a_{i}$ depends only on the coordinate $\eta_{i}$, the argument of the left-hand side of the above equality is a local function depending on the coordinates of the box $\Lambda_{N}$. Hence, we have,

$$
\sum_{i \in \Lambda_{N}} \int \frac{a_{i}}{2} \frac{v_{N}}{u_{N}}\left(\frac{\partial\left(u_{N} / v_{N}\right)}{\partial \eta_{i}}\right)^{2} v_{N} \mathrm{~d} m=\sum_{i \in \Lambda_{N}} \int \frac{a_{i}}{2} \frac{v_{N}}{u_{N}}\left(\frac{\partial\left(u_{N} / v_{N}\right)}{\partial \eta_{i}}\right)^{2} \mathrm{~d} \nu
$$

Combining this with (2) we conclude that,

$$
\frac{1}{2} \sum_{i \in \Lambda_{N}} \int a_{i} \frac{v_{N}}{u_{N}}\left(\frac{\partial\left(u_{N} / v_{N}\right)}{\partial \eta_{i}}\right)^{2} \mathrm{~d} \nu=\sum_{i \in \delta \Lambda_{N}} \int b_{i} \frac{v_{N}}{u_{N}} \frac{\partial\left(u_{N} / v_{N}\right)}{\partial \eta_{i}}\left(u_{\Lambda_{N, i}}-u_{N} v_{\delta, N, i}\right) \mathrm{d} m
$$

At this point, let us note, using Cauchy-Schwartz inequality twice that the right-hand side of (3) can be upper bounded as follows:

$$
\begin{aligned}
\left|\sum_{i \in \delta \Lambda_{N}} \int b_{i} \frac{v_{N}}{u_{N}} \frac{\partial\left(u_{N} / v_{N}\right)}{\partial \eta_{i}}\left(u_{\Lambda_{N, i}}-u_{N} v_{\delta, N, i}\right) \mathrm{d} m\right| & \leq 2 B \sum_{i \in \delta \Lambda_{N}} \int \frac{v_{N}}{u_{N}}\left|\frac{\partial\left(u_{N} / v_{N}\right)}{\partial \eta_{i}}\right| \mathrm{d} \mu \\
& \leq 2 B\left|\delta \Lambda_{N}\right|^{1 / 2} \sqrt{\sum_{i \in \delta \Lambda_{N}} \int \frac{v_{N}}{u_{N}}\left(\frac{\partial\left(u_{N} / v_{N}\right)}{\partial \eta_{i}}\right)^{2} \mathrm{~d} \nu}
\end{aligned}
$$


where $B:=\sup _{i, \eta}\left|b_{i}(\eta)\right|$. On the other hand, the left-hand side of equality (3) can be lower bounded using the fact that $a:=\inf _{i, \eta} a_{i}(\eta)>0$, so that we conclude that,

$$
a \frac{1}{2} \sum_{i \in \Lambda_{N}} \int \frac{v_{N}}{u_{N}}\left(\frac{\partial\left(u_{N} / v_{N}\right)}{\partial \eta_{i}}\right)^{2} \mathrm{~d} \nu \leq U_{N} \sqrt{\sum_{i \in \delta \Lambda_{N}} \int \frac{v_{N}}{u_{N}}\left(\frac{\partial\left(u_{N} / v_{N}\right)}{\partial \eta_{i}}\right)^{2} \mathrm{~d} \nu}
$$

where, $U_{N}:=2 B d(4 R)^{d / 2} N^{(d-1) / 2}$. At this point let us define for $n \geq 1, c_{n}:=\sum_{i \in \delta \Lambda_{n}} \int \frac{v_{n}}{u_{n}}\left(\frac{\partial\left(u_{n} / v_{n}\right)}{\partial \eta_{i}}\right)^{2} \mathrm{~d} \nu$. It follows from the inequality $c_{n} \leq \frac{1}{2} \sum_{i \in \Lambda_{n}} \int \frac{v_{n}}{u_{n}}\left(\frac{\partial\left(u_{n} / v_{n}\right)}{\partial \eta_{i}}\right)^{2} \mathrm{~d} \nu$, the fact that $a>0$ and inequality (4), that there is some constant $K>0$ such that,

$$
c_{n} \leq K n^{d-1}
$$

for every $n \geq 1$. We will complete the proof of Theorem 1 separately for each part. Before, we need to state a convexity property for the free energy, which will turn out to be crucial (see Lem. 3.3 of [2] for a similar statement). Let $x, y \in T$ and $f(x, y): T^{2} \rightarrow \mathbf{R}$ be bounded, and for each $y$ a measurable function of $x$. For each $x, y \in T$ assume that the partial derivative $f_{y}:=\partial f / \partial y$ exists and is bounded in $T^{2}$. Then if $\alpha(x): T \rightarrow$ $[0, \infty)$ is a bounded measurable function with $\int_{T} \alpha \mathrm{d} x=1$ we have, $\left(\frac{\left(\int_{T} \alpha f \mathrm{~d} x\right)_{y}}{\int_{T} \alpha f \mathrm{~d} x}\right)^{2} \int_{T} \alpha f \mathrm{~d} x \leq \int_{T} \alpha\left(\frac{f_{y}}{f}\right)^{2} f \mathrm{~d} x$, where $\left(\int_{T} \alpha f \mathrm{~d} x\right)_{y}$ is the partial derivative of $\int_{T} \alpha f \mathrm{~d} x$ with respect to $y$. It now follows from this property and the fact that $\nu$ is a product measure with smooth densities on boxes that for any $i, F, N$ such that $i \in F \subset \Lambda_{N}$,

$$
\int \frac{u_{F}}{v_{F}}\left(\frac{\partial\left(u_{F} / v_{F}\right)}{\partial \eta_{i}}\right)^{2} \mathrm{~d} \nu \leq \int \frac{u_{N}}{v_{N}}\left(\frac{\partial\left(u_{N} / v_{N}\right)}{\partial \eta_{i}}\right)^{2} \mathrm{~d} \nu
$$

\subsection{Proof of part (i)}

Assume that $\mu$ is translation invariant. Substituting (5) in (4) we conclude that

$$
a \frac{1}{2} \sum_{i \in \Lambda_{N}} \int \frac{v_{N}}{u_{N}}\left(\frac{\partial\left(u_{N} / v_{N}\right)}{\partial \eta_{i}}\right)^{2} \mathrm{~d} \nu \leq C_{1} N^{d-1}
$$

for some constant $C_{1}>0$. Now, let $F$ be a bounded subset of the lattice and $i \in F$. By the convexity property (6) we have that,

$$
\sum_{j \in \Lambda_{N}^{F}} \int \frac{u_{F_{i-j}}}{v_{F_{i-j}}}\left(\frac{\partial\left(u_{F_{i-j}} / v_{F_{i-j}}\right)}{\partial \eta_{j}}\right)^{2} \mathrm{~d} \nu \leq \sum_{j \in \Lambda_{N}^{F}} \int \frac{u_{N}}{v_{N}}\left(\frac{\partial\left(u_{N} / v_{N}\right)}{\partial \eta_{j}}\right)^{2} \mathrm{~d} \nu \leq \sum_{j \in \Lambda_{N}} \int \frac{u_{N}}{v_{N}}\left(\frac{\partial\left(u_{N} / v_{N}\right)}{\partial \eta_{j}}\right)^{2} \mathrm{~d} \nu
$$

where for $k \in \mathbf{Z}^{d}$, we define $F_{k}=F-k$. Finally, the translation invariance of $\mu$ and $\nu$ imply that $u_{F_{k}} / v_{F_{k}}=$ $u_{F} / v_{F}$, so that we can deduce that,

$$
\left|\Lambda_{N}^{F}\right| \int \frac{u_{F}}{v_{F}}\left(\frac{\partial\left(u_{F} / v_{F}\right)}{\partial \eta_{i}}\right)^{2} \mathrm{~d} \nu \leq C_{1} N^{d-1}
$$

where $C_{1}$ is some constant and $\Lambda_{N}^{F}$ are the points of $\Lambda_{N}$ at a distance larger than $\operatorname{diam}(F)$ to $\delta \Lambda_{N}$. Then, we can apply (6) to each $j \in \Lambda_{N}^{F}$ and use the the translation invariance of $\mu$ and $\nu$ to conclude that 
$\frac{a}{2}\left|\Lambda_{N}^{F}\right| \int \frac{u_{F}}{v_{F}}\left(\frac{\partial\left(u_{F} / v_{F}\right)}{\partial \eta_{i}}\right)^{2} \mathrm{~d} m \leq C_{2} N^{d-1}$, where $C_{2}$ is a constant. Now, note that for $N$ big enough, $\left|\Lambda_{N}^{F}\right| \geq C_{2} N^{d}$ for some constant $C_{2}>0$. Hence we have that

$$
\int \frac{u_{F}}{v_{F}}\left(\frac{\partial\left(u_{F} / v_{F}\right)}{\partial \eta_{i}}\right)^{2} \mathrm{~d} m \leq C_{2} N^{-1}
$$

Letting $N \rightarrow \infty$ we conclude that $\int \frac{u_{F}}{v_{F}}\left(\frac{\partial\left(u_{F} / v_{F}\right)}{\partial \eta_{i}}\right)^{2} \mathrm{~d} m=0$. The smoothness and positivity of $u_{F}$ and $v_{F}$ now imply that for every bounded $F \subset \mathbf{Z}^{d}$ and $i \in F$, it is true that $\frac{\partial\left(u_{F} / v_{F}\right)}{\partial \eta_{i}}=0$. This implies that $\mu=\nu$.

\subsection{Proof of part (ii)}

Let us note that by the convexity property (6), we have $c_{n} \leq c_{m}$, whenever $n \leq m$. Hence, by inequality (4) we can conclude that $\sum_{n=1}^{N} c_{n} \leq C_{3} N^{\frac{d-1}{2}} \sqrt{c_{N}}$, for some constant $C_{3}>0$. An application of Lemma 5.4 of Holley-Stroock [2] shows that $c_{n}=0$ for $n \geq 1$ and $d=1$ or 2. This implies that $\mu=\nu$.

\subsection{Proof of part (iii)}

The following lemma contains the main argument of the proof:

Lemma 1. Suppose that $\nu$ is the Lebesgue measure. Then for every $m \in \mathbf{Z}$ we have,

$$
c_{n+m} \geq \frac{c_{m}}{C}\left(1+\frac{1}{C}\right)^{n-1} \quad n \geq 1
$$

where $C=B\left((2 R+1)^{d}+1\right)^{2} / a, B=\sup _{i, \eta}\left|b_{i}(\eta)\right|$ and $a=\inf _{i, \eta} a_{i}(\eta)$.

Before proving this lemma we will show how part (iii) of Theorem 1 is implied by Lemma 1 and inequality (5). Assume that there is an $m \geq 1$ such that $c_{m}>0$. Then Lemma 1 implies that the sequence $c_{n+m}$ is bounded from below by an exponentially growing function in $n$. But this contradicts the polynomial upper bound of inequality (5). Thus, $\frac{\partial u_{N}}{\partial \eta_{i}}=0$ whenever $i \in \Lambda_{N}$ and $N \geq 1$.

In what follows we prove Lemma 1. Define $\delta \lambda_{N, i}:=\Lambda_{N, i} \backslash \Lambda_{N}$ as the subset of $\delta \Lambda_{N+1}$ contained in the ball $B_{i}$. Then,

$$
\left|\int b_{i} \frac{1}{u_{N}}\left(\frac{\partial u_{N}}{\partial \eta_{i}}\right)\left(u_{\Lambda_{N, i}}-u_{N}\right) \mathrm{d} m\right| \leq \int \frac{1}{u_{N}}\left|\frac{\partial u_{N}}{\partial \eta_{i}}\right|\left|\int b_{i}\left(u_{\Lambda_{N, i}}-u_{N}\right) \mathrm{d} m_{\delta \lambda_{N, i}}\right| \mathrm{d} m_{\Lambda_{N}} .
$$

Let us now call $M_{N, i}$ the cardinality of the set $\delta \lambda_{N, i}$. We will label the elements of this set by $j_{1}, j_{2}, \ldots, j_{M_{N, i}}$. Next, we define a sequence of sets $\Lambda_{N} \subseteq F_{i, N, k} \subseteq \Lambda_{N, i}$, for $0 \leq k \leq M_{N, i}$, recursively as $F_{i, N, k+1}=F_{i, N, k} \cup$ $\left\{j_{k+1}\right\}$, where $F_{i, N, 0}=\Lambda_{N}$. Note that $F_{i, N, M_{N, i}}=\Lambda_{N, i}$. Then,

$$
\left|\int b_{i}\left(u_{\Lambda_{N, i}}-u_{N}\right) \mathrm{d} m_{\delta \lambda_{N, i}}\right| \leq \sum_{k=0}^{M_{N, i}-1}\left|\int b_{i}\left(u_{F_{i, N, k+1}}-u_{F_{i, N, k}}\right) \mathrm{d} m_{F_{i, N, k+1} \backslash \Lambda_{N}}\right| .
$$

Now, since $\int u_{F_{i, N, k+1}} \mathrm{~d} m_{\left\{j_{k+1}\right\}}=u_{F_{i, N, k}}$, we can use Cauchy-Schwartz inequality to deduce that,

$$
\left|\int b_{i}\left(u_{F_{i, N, k+1}}-u_{F_{i, N, k}}\right) \mathrm{d} m_{F_{i, N, k+1} \backslash \Lambda_{N}}\right| \leq B \sqrt{u_{N}}\left(\int \frac{1}{u_{F_{i, N, k+1}}}\left(\frac{\partial u_{F_{i, N, k+1}}}{\partial \eta_{j_{k+1}}}\right)^{2} \mathrm{~d} m_{F_{i, N, k+1} \backslash \Lambda_{N}}\right)^{1 / 2} .
$$


Using the bound (10) on (9) and substituting this estimate for the right hand side of (8), we can see that

$$
\begin{aligned}
\left|\int b_{i} \frac{1}{u_{N}}\left(\frac{\partial u_{N}}{\partial \eta_{i}}\right)\left(u_{\Lambda_{N, i}} u_{N}\right) \mathrm{d} m\right| \leq & B \sum_{k=0}^{M_{N, i}-1}\left(\frac{1}{2} \int \frac{1}{u_{N}}\left(\frac{\partial u_{N}}{\partial \eta_{i}}\right)^{2} \mathrm{~d} m_{\Lambda_{N}}\right. \\
& \left.+\frac{1}{2} \int \frac{1}{u_{F_{i, N, k+1}}}\left(\frac{\partial u_{F_{i, N, k+1}}}{\partial \eta_{j_{k+1}}}\right)^{2} \mathrm{~d} m_{F_{i, N, k+1}}\right) \\
\leq & \frac{B}{2} \sum_{k=0}^{M_{N, i}} \int \frac{1}{u_{F_{i, N, k}}}\left(\frac{\partial u_{F_{i, N, k}}}{\partial \eta_{j_{k}}}\right)^{2} \mathrm{~d} m_{F_{i, N, k}}
\end{aligned}
$$

Now, bounding the absolute value of the right hand side of (3) by the above estimate and remarking that $M_{N, i} \leq(2 R+1)^{d}$, we conclude that,

$$
\begin{aligned}
a \sum_{i \in \Lambda_{N}} \int \frac{1}{u_{N}^{2}}\left(\frac{\partial u_{N}}{\partial \eta_{i}}\right)^{2} \mathrm{~d} \mu & \leq B \sum_{k=0}^{(2 R+1)^{d}} \sum_{i \in \delta \Lambda_{N}} \theta_{M_{N, i}}(k) \int \frac{1}{u_{F_{i, N, k}}}\left(\frac{\partial u_{F_{i, N, k}}}{\partial \eta_{j_{k}}}\right)^{2} \mathrm{~d} m_{\Lambda_{N+1}} \\
& \leq B\left((2 R+1)^{d}+1\right)^{2} \sum_{i \in \delta \Lambda_{N+1}} \int \frac{1}{u_{N+1}^{2}}\left(\frac{\partial u_{N+1}}{\partial \eta_{i}}\right)^{2} \mathrm{~d} \mu
\end{aligned}
$$

where $\theta_{y}(x): \mathbf{Z} \rightarrow\{0,1\}$ equals 0 if $x>y$ and 1 otherwise, and we have used the convexity property (6) in the second inequality. Finally, from (11) and using again the convexity (6) we conclude that $\sum_{n=1}^{N} c_{n} \leq C c_{N+1}$, where $C=B\left((2 R+1)^{d}+1\right)^{2} / a$. In turn, this inequality implies that $\sum_{n=1}^{N-1} c_{n-1+m} \leq C c_{N-1+m}$ for $m \geq 1$, $N \geq 2$. Now, it is true that $c_{n-1+m} \geq \bar{c}_{n}$ where $\bar{c}_{n}$ is the unique solution of the system of equations $\sum_{n=1}^{N-1} \bar{c}_{n}=$ $C \bar{c}_{N}, N \geq 2$, with initial condition $\bar{c}_{1}=c_{m}$. The lemma follows from the fact that $\bar{c}_{n}=\frac{c_{m}}{C}\left(1+\frac{1}{C}\right)^{n-2}$, for $n \geq 2$.

\section{INFINITE PARTICLE SPIN SYSTEMS}

Let us now prove Theorem 2. The proof captures the main ideas of the proofs of part (i) and (ii) of Theorem 1 in an infinite particle system context. We will keep the notation introduced in the previous section so that $\Lambda_{N}:=[-N R, N R]^{d} \cap \mathbf{Z}^{d}$. So let $\phi$ be any local function depending only on coordinates on $\Lambda_{N}$. Then, from the invariance of the measure $\nu$, we have that,

$$
0=\int L \phi \mathrm{d} \nu=\sum_{x \in \Lambda_{N-1}} \int_{\Lambda_{N}} c(x, \eta)\left(\phi\left(\eta^{x}\right)-\phi(\eta)\right) \mathrm{d} \nu_{N}+\sum_{x \in \delta \Lambda_{N}} \int_{\Lambda_{N+1}} c(x, \eta)\left(\phi\left(\eta^{x}\right)-\phi(\eta)\right) \mathrm{d} \nu_{N+1} .
$$

Now, let $\mu$ be some arbitrary invariant measure. As in the proof of Lemma 1.16 of Holley-Stroock [1], it is possible to conclude that the ellipticity condition on the flip rates implies that the restriction of any invariant measure to finite boxes is equivalent to the uniform measure on the corresponding box. Thus, we can choose $\phi(\eta):=\ln \frac{u_{N}}{v_{N}}$ in $(12)$ to conclude that,

$$
0=\sum_{x \in \Lambda_{N-1}} \int_{\Lambda_{N}} c(x, \eta) \ln \frac{\phi_{N}\left(\eta^{x}\right)}{\phi_{N}(\eta)} \mathrm{d} \mu_{N}+\sum_{x \in \delta \Lambda_{N}} \int_{\Lambda_{N+1}} c(x, \eta) \ln \frac{\phi_{N}\left(\eta^{x}\right)}{\phi_{N}(\eta)} \mathrm{d} \mu_{N+1}
$$


Now, the invariance of $\nu$ implies that $\sum_{x \in \Lambda_{N}} \int_{\Lambda_{N}} c(x, \eta)\left(\phi_{N}\left(\eta^{x}\right)-\phi_{N}(\eta)\right) \mathrm{d} \nu_{N}=0$. Combining this with equality (13), we then have,

$$
\begin{aligned}
0= & -\sum_{x \in \Lambda_{N-1}} \int_{\Lambda_{N}} c(x, \eta) \phi_{N}^{x} F\left(\frac{\phi_{N}}{\phi_{N}^{x}}\right) \mathrm{d} \nu_{N}+\sum_{x \in \delta \Lambda_{N}} \int_{\Lambda_{N+1}} c(x, \eta) \ln \frac{\phi_{N}^{x}}{\phi_{N}} \mathrm{~d} \mu_{N+1} \\
& +\sum_{x \in \delta \Lambda_{N}} \int_{\Lambda_{N+1}} c(x, \eta)\left(\phi_{N}^{x}-\phi_{N}\right) \mathrm{d} \nu_{N+1},
\end{aligned}
$$

where we have introduced the notation $\phi_{N}^{x}(\eta):=\phi_{N}\left(\eta^{x}\right)$ and defined for $x>0$ the function $F(x):=x \ln x+1-x$. Now, adding and subtracting $\sum_{x \in \delta \Lambda_{N}} \int_{\Lambda_{N}} c(x, \eta) \phi_{N}^{x} F\left(\frac{\phi_{N}}{\phi_{N}^{x}}\right) \mathrm{d} \nu_{N}$ to the right-hand side of (14) we then have that,

$$
\begin{aligned}
0= & -\sum_{x \in \Lambda_{N}} \int_{\Lambda_{N}} c(x, \eta) \phi_{N}^{x} F\left(\frac{\phi_{N}}{\phi_{N}^{x}}\right) \mathrm{d} \nu_{N}+\sum_{x \in \delta \Lambda_{N}} \int_{\Lambda_{N+1}} c(x, \eta) \ln \frac{\phi_{N}^{x}}{\phi_{N}} \mathrm{~d} \mu_{N+1} \\
& +\sum_{x \in \delta \Lambda_{N}} \int_{\Lambda_{N+1}} c(x, \eta)\left(\phi_{N}^{x}-\phi_{N}\right) \mathrm{d} \nu_{N+1}+\sum_{x \in \delta \Lambda_{N}} \int_{\Lambda_{N}} c(x, \eta) \ln \frac{\phi_{N}^{x}}{\phi_{N}} \mathrm{~d} \mu_{N}+\sum_{x \in \delta \Lambda_{N}} \int_{\Lambda_{N}} c(x, \eta)\left(\phi_{N}^{x}-\phi_{N}\right) \mathrm{d} \nu_{N} .
\end{aligned}
$$

Now, let us note that for $x>0$ it is true that $F(x) \geq \frac{1}{2}(1-\sqrt{x})^{2}$. It then follows from the previous calculation that,

$$
\begin{aligned}
\frac{1}{2} \sum_{x \in \Lambda_{N}} \int_{\Lambda_{N}} c(x, \eta) \phi_{N}^{x}\left(\sqrt{\phi_{N}^{x}}-\sqrt{\phi_{N}}\right)^{2} \mathrm{~d} \nu_{N} \leq & \sum_{x \in \delta \Lambda_{N}} \int_{\Lambda_{N+1}} c(x, \eta) \ln \frac{\phi_{N}^{x}}{\phi_{N}} \mathrm{~d} \mu_{N+1} \\
& +\sum_{x \in \delta \Lambda_{N}} \int_{\Lambda_{N+1}} c(x, \eta)\left(\phi_{N}-\phi_{N}^{x}\right) \mathrm{d} \nu_{N+1} . \\
& +\sum_{x \in \delta \Lambda_{N}} \int_{\Lambda_{N}} c(x, \eta) \ln \frac{\phi_{N}^{x}}{\phi_{N}} \mathrm{~d} \mu_{N} \\
& +\sum_{x \in \delta \Lambda_{N}} \int_{\Lambda_{N}} c(x, \eta)\left(\phi_{N}^{x}-\phi_{N}\right) \mathrm{d} \nu_{N} .
\end{aligned}
$$

On the other hand,

$$
\begin{aligned}
\sum_{x \in \delta \Lambda_{N}} \int_{\Lambda_{N+1}} c(x, \eta) \ln \frac{\phi_{N}^{x}}{\phi_{N}} \mathrm{~d} \mu_{N+1} & \leq \sum_{x \in \delta \Lambda_{N}} \int_{\eta \in \Lambda_{N+1}: \frac{\phi_{N}^{x}}{\phi_{N}} \geq 1} c(x, \eta) \ln \frac{\phi_{N}^{x}}{\phi_{N}} \mathrm{~d} \mu_{N+1} \\
& \leq \bar{c} \sum_{x \in \delta \Lambda_{N}} \int \phi_{N} \ln \frac{\phi_{N}^{x}}{\phi_{N}} \mathrm{~d} \nu_{N} \leq \bar{c} \sum_{x \in \delta \Lambda_{N}} \int_{\Lambda_{N+1}}\left|\phi_{N}^{x}-\phi_{N}\right| \mathrm{d} \nu_{N},
\end{aligned}
$$

where we have defined $\bar{c}:=\sup _{x, \eta} c(x, \eta)$. Similarly, $\sum_{x \in \delta \Lambda_{N}} \int_{\Lambda_{N}} c(x, \eta) \ln \frac{\phi_{N}^{x}}{\phi_{N}} \mathrm{~d} \mu_{N} \leq \bar{c} \sum_{x \in \delta \Lambda_{N}} \int_{\Lambda_{N+1}} \mid \phi_{N}^{x}-$ $\phi_{N} \mid \mathrm{d} \nu_{N}$. Therefore, substituting these estimates back into inequality (15), we see that,

$$
\frac{c}{2} \sum_{x \in \Lambda_{N}} \int_{\Lambda_{N}}\left(\sqrt{\phi_{N}^{x}}-\sqrt{\phi_{N}}\right)^{2} \mathrm{~d} \nu_{N} \leq 4 \bar{c} \sum_{x \in \delta \Lambda_{N}} \int_{\Lambda_{N+1}}\left|\phi_{N}^{x}-\phi_{N}\right| \mathrm{d} \nu_{N}
$$

where $\underline{c}:=\inf _{x, \eta} c(x, \eta)$. Let us now call $\nu_{x}$ the marginal of the product measure $\nu$ at site $x$. Note that $\nu_{x}$ is an invariant measure for the two state Markov chain with a generator acting on functions $f$ of $\eta_{x}$ as $L_{x} f\left(\eta_{x}\right):=$ 
$\bar{c}\left(x, \eta_{x}\right)\left(f\left(1-\eta_{x}\right)-f\left(\eta_{x}\right)\right)$, with $\bar{c}\left(x, \eta_{x}\right):=\int c(x, \eta) \Pi_{y \neq x} \mathrm{~d} \nu_{y}$. By the ellipticity hypothesis, we have that $\inf _{x, \eta_{x}} \bar{c}\left(x, \eta_{x}\right)>0$. It follows that $\underline{v}:=\inf _{x \in \mathbf{Z}^{d}}\left\{\nu_{x}\left(\eta_{x}=1\right), \nu_{x}\left(\eta_{x}=0\right)\right\}>0$. We then have, using CauchySchwartz inequality and the fact that $(\sqrt{x}+\sqrt{y})^{2} \leq 2(x+y)$ for $x \geq 0$ and $y \geq 0$.

$$
\begin{aligned}
\int_{\Lambda_{N+1}}\left|\phi_{N}^{x}-\phi_{N}\right| \mathrm{d} \nu_{N} & \leq 2\left(\int_{\Lambda_{N+1}}\left(\sqrt{\phi_{N}^{x}}-\sqrt{\phi_{N}}\right)^{2} \mathrm{~d} \nu_{N}\right)^{1 / 2}\left(\int_{\Lambda_{N+1}}\left(\phi_{N}^{x}+\phi_{N}\right) \mathrm{d} \nu_{N}\right)^{1 / 2} \\
& \leq 2\left(1+\frac{1}{\underline{v}}\right)\left(\int_{\Lambda_{N}}\left(\sqrt{\phi_{N}^{x}}-\sqrt{\phi_{N}}\right)^{2} \mathrm{~d} \nu_{N}\right)^{1 / 2}
\end{aligned}
$$

Substituting (17) in inequality (16) and using Cauchy-Schwartz inequality again we finally obtain the following relationship,

$$
\sum_{x \in \Lambda_{N}} \int_{\Lambda_{N}}\left(\sqrt{\phi_{N}^{x}}-\sqrt{\phi_{N}}\right)^{2} \mathrm{~d} \nu \leq V_{N} \sqrt{\sum_{x \in \delta \Lambda_{N}} \int_{\Lambda_{N}}\left(\sqrt{\phi_{N}^{x}}-\sqrt{\phi_{N}}\right)^{2} \mathrm{~d} \nu}
$$

where $V_{N}:=C \sqrt{\left|\delta \Lambda_{N}\right|}=C d(4 R)^{d / 2} N^{(d-1) / 2}$ with $C:=\frac{8 \bar{c}(1+\underline{\underline{1}})}{\underline{c}}$. At this point we define as in the previous section for $n \geq 1$, the quantities, $c_{n}:=\sum_{x \in \delta \Lambda_{n}} \int_{\Lambda_{n}}\left(\sqrt{\phi_{n}^{x}}-\sqrt{\phi_{n}}\right)^{2} \mathrm{~d} \nu$. Clearly, there is some constant $K>0$ such that,

$$
c_{n} \leq C n^{d-1}
$$

for every $n \geq 1$. Let us now remark the following convexity property, analogous to the convexity inequality (6) for diffusions, satisfied for any $x, F, N$ such that $x \in F \subset \Lambda_{N}$,

$$
\int\left(\sqrt{\frac{u_{F}\left(\eta^{x}\right)}{v_{F}\left(\eta^{x}\right)}}-\sqrt{\frac{u_{F}(\eta)}{v_{F}(\eta)}}\right)^{2} \mathrm{~d} \nu \leq \int\left(\sqrt{\frac{u_{N}\left(\eta^{x}\right)}{v_{N}\left(\eta^{x}\right)}}-\sqrt{\frac{u_{N}(\eta)}{v_{N}(\eta)}}\right)^{2} \mathrm{~d} \nu
$$

A combination of inequalities $(18,19)$ and $(20)$ now enable us to prove Theorem 2 . The details are as those of the proof of parts (i) and (ii) of Theorem 1.

\section{REFERENCES}

[1] R. Holley and D. Stroock, In one and two dimensions, every stationary measure for a stochastic Ising model is a Gibbs state. Commun. Math. Phys. 55 (1977) 37-45.

[2] R. Holley and D. Stroock, Diffusions on an Infinite Dimensional Torus. J. Funct. Anal. 42 (1981) 29-63.

[3] H. Kunsch, Non reversible stationary measures for infinite interacting particle systems. Z. Wahrsch. Verw. Gebiete 66 (1984) 407-424.

[4] T.M. Liggett, Interacting Particle Systems. Springer-Verlag, New York (1985).

[5] T.S. Mountford, A Coupling of Infinite Particle Systems. J. Math. Kyoto Univ. 35 (1995) 43-52.

[6] A.F. Ramírez, An elementary proof of the uniqueness of invariant product measures for some infinite dimensional diffusions. C. R. Acad. Sci. Paris Sér. I Math. (to appear).

[7] A.F. Ramírez, Relative Entropy and Mixing Properties of Infinite Dimensional Diffusions. Probab. Theory Related Fields 110 (1998) 369-395.

[8] A.F. Ramírez and S.R.S. Varadhan, Relative Entropy and Mixing Properties of Interacting Particle Systems. J. Math. Kyoto Univ. 36 (1996) 869-875. 